\title{
Philosophiques
}

\section{Leibniz et les hypothèses de physique}

\section{François Duchesneau}

Volume 9, numéro 2, octobre 1982

URI : https://id.erudit.org/iderudit/203193ar

DOI : https://doi.org/10.7202/203193ar

Aller au sommaire du numéro

Éditeur(s)

Société de philosophie du Québec

ISSN

0316-2923 (imprimé)

1492-1391 (numérique)

Découvrir la revue

Citer cet article

Duchesneau, F. (1982). Leibniz et les hypothèses de physique. Philosophiques, 9(2), 223-238. https://doi.org/10.7202/203193ar

\section{Résumé de l'article}

Dans la période 1671-1678, Leibniz met au point une conception des hypothèses de physique qui les subordonne à un double critère de validité. Il s'agit en premier lieu de validité heuristique. Mais l'hypothèse doit aussi comporter une justification analytique dont la possibilité tiendrait à une transcription géométrique des séquences de phénomènes. Il importe de déterminer comment validité heuristique et validité démonstrative se concilient. d'utilisation que vous pouvez consulter en ligne.

https://apropos.erudit.org/fr/usagers/politique-dutilisation/ 


\title{
LEIBNIZ ET LES HYPOTHÈSES DE PHYSIQUE
}

\author{
par François Duchesneau
}

\begin{abstract}
RÉSUMÉ. Dans la période 1671-1678, Leibniz met au point une conception des hypothèses de physique qui les subordonne à un double critère de validité. Il s'agit en premier lieu de validité heuristique. Mais l'hypothèse doit aussi comporter une justification analytique dont la possibilité tiendrait à une transcription géométrique des séquences de phénomènes. Il importe de déterminer comment validité heuristique et validité démonstrative se concilient.
\end{abstract}

ABSTRACT. In the period 1671-1678, Leibniz works out his conception of hypothesis in Physics with reference to a twofold validity criterion. Heuristic validity applies. But hypotheses must also involve an analytic justification which might be achieved through geometrically transcribing the sequences of phenomena. One has to determine how Leibniz accords heuristic with demonstrative validation.

Le dessein de l'analyse que je vous propose est limité. Vous m'accorderez sans doute que le système leibnizien intègre une réflexion sur la connaissance scientifique dans certaines de ses productions significatives: calcul infinitésimal, dynamique, logique symbolique. Le système intègre cette réflexion aux fins d'une métaphysique et d'une morale dont l'épistémologue a quelque difficulté à faire son profit. La synthèse leibnizienne est trop «symphonique» pour qu'on y démêle aisément les concepts d'une théorie de la science. L'analyse s'y perd dans des renvois en harmoniques, et l'on est vite déçu des apparentes inconséquences d'une réflexion épistémologique qui prend et ne prend pas à la fois la science pour objet. Pourtant, il doit y avoir moyen de satisfaire la curiosité de celui qui cherche dans Leibniz les éléments d'analyse de la science leibnizienne telle qu'elle se développe par exemple dans l'Unicum opticae, catoptricae et dioptricae principium ou dans le Specimen dynamicum. Mettant entre parenthèses la perspec- 
tive hégémonique du système, n'est-il pas possible de suivre certains filons dans l'inventaire des ressources leibniziennes ad usum $\epsilon \pi \iota \sigma \tau \eta \mu о \nu \iota \kappa \eta s$ rationis? Je vais tenter de suivre avec vous l'un de ces filons: il s'agit de la doctrine des hypothèses de physique qui émerge à la période cruciale où Leibniz révise la mécanique cartésienne et la philosophie naturelle (physique) des Principia de Descartes, période qui s'étend de l'Hypothesis pbysica nova (1671) à la formulation initiale des principes de la dynamique 1678$)^{1}$.

La correspondance de Leibniz avec Conring établit des jalons significatifs sur la conception leibnizienne des hypothèses. Dans un premier temps, il nous semble utile de relever ces repères essentiels et d'en inférer, si possible, comment se transforment les normes épistémologiques impliquées.

Conring juge que rien de neuf en matière de doctrine n'a pu être établi par l'experimental philosophy ${ }^{2}$. Par négligence du mode déductif de développement, le savoir que les expérimentateurs et observateurs de la Royal Society tentent d'élaborer, ne peut s'élever au rang de science. Cette critique est certes d'un aristotélicien qui ne saurait admettre le caractère révisable des explications inférées des données de fait relatives aux phénomènes; mais Conring insiste en fait de façon toute particulière sur l'apriorité des prémisses ultimes sur lesquelles doit se construire la philosophie de la nature. Nous n'avons pas d'aperçu clair sur la façon dont Conring envisage l'instauration de cette philosophie de la nature. Sans doute lui accorderait-il un statut démonstratif, pourvu que les raisons invoquées pour rendre compte des phénomènes s'avèrent conformes aux principes métaphysiques du système aristotélicien. Leibniz réplique en formulant une thèse intéressante sur la nature de la démonstration de science et sur le

1. Cf. Yvon Belaval, "Premières Animadversions sur les 'Principes' de Descartes», in Études leibniziennes, Paris: 'Gallimard, 1976, p. 58 n. 8.

2. Cf. Conring an Leibniz, 16/26 fév. 1671. P (Die Pbilosopbischen Scbriften, éd. Gerhardt), I, pp. 171-172. 
statut d'hypothèse dévolu aux explications empiriques. La disparité de statut de la démonstration-modèle par rapport à l'hypothèse constitue le problème épistémologique fondamental pour la critique leibnizienne.

Leibniz propose comme formule de la démonstration la structure d'une chaîne de définitions (catena definitionum), ce qui ramène la logique de la démonstration à l'opération de substitution des équivalents. Cette figure de la démonstration est nettement signalée entre autres par Couturat ${ }^{3}$ et Rescher ${ }^{4}$, et elle est conforme à la typologie des idées que développeront les Meditationes (1684) et le Discours de métaphysique $(1686)^{5}$. De cette typologie des idées, on pourrait d'ailleurs inférer quelles conditions liminales déterminent l'établissement de concepts scientifiques aptes à se modeler sur le processus de la substitution des équivalents. Dans la réplique à Conring, l'hypothèse est identifiée à une anticipation de détermination causale, lorsque l'analyse des phénomènes n'est restée que partielle. L'hypothèse doit rendre compte de tous les phénomènes pertinents en achevant pour ainsi dire l'investigation «nominale» des causes probables; comme un mécanisme d'horlogerie dont le mode interne d'opération ne nous est pas connu, mais dont nous fournissons la raison suffisante en projetant mentalement un système de rouages et de structures aptes à accomplir un effet similaire. Mais les mêmes phénomènes artificiels pourraient sans doute être obtenus suivant divers procédés; seule la décomposition analytique du mécanisme peut nous révéler sans équivoque possible le procédé utilisé. Cela

3. Cf. Louis Couturat, La logique de Leibniz (1901), Hildesheim: Olms, 1969, p. 206: «Toute démonstration consiste à substituer la définition au défini, c'est-à-dire à remplacer un terme (complexe) par un groupe de termes (plus simples) qui lui est équivalent. Ainsi le fondement essentiel de la déduction est le principe de la substitution des équivalents. C'est lui qui est le principe suprême et unique de la Logique, et non pas le principe du syllogisme (le Dictum de omni et nullo d'Aristote, car celui-ci n'est pas, comme on le croit souvent, un axiome identique, mais un théorème qui se démontre au moyen du principe précédent». Cf. lettre à Placcius du 16 novembre 1686: "Quanquam enim mihi non alia ibi videatur opus esse demonstratione, quam quae pendet ex mutua aequipollentium substitutione...» (cité par Couturat, p. 206 n. 2) et lettre à Vagetius du 10 janvier 1687: «Possumus etiam omnes praedicationes reducere ad aequipollentias, supplendo aliquid ad complendam reciprocationem; unde rursus apparet propositum" (cité par Couturat, p. 206 n. 3).

4. Nicholas Rescher, Leibniz. An Introduction to his Philosopby. Oxford: Blackwell, 1969, p. 22: "Leibnizian analysis is a logical process of a very rudimentary sort, based on the inferential procedures of definitional replacement and determination of predicational containment through explicit use of logical processes of inference".

5. Cf, P, IV, pp. $422-424$, et $\$ 24$, pp. $449-450$. 
correspondrait à fournir une définition du processus générateur des effets phénoménaux: «Ipsum praecise modum quo Artifex usus est, nisi dissoluto opere definire non possum» ${ }^{6}$. Évidemment, lorsqu'il s'agit non plus des machines de l'art, mais des machines de la nature, dont la complexité se déploie à l'infini dans la décomposition analytique, l'hypothèse ne peut que figurer l'ordre causal. Mais il est significatif que Leibniz ne limite pas cette figuration à fournir la raison suffisante des expériences actuellement disposibles: il réclame de l'hypothèse qu'elle fournisse le projet architectonique d'autres effets possibles que l'expérience aura à charge de révéler dans une investigation plus systématique et plus complète des phénomènes ${ }^{7}$.

En fonction de cette doctrine de l'hypothèse, Leibniz accrédite les investigations empiriques (compilation et corrélation de données suivant la méthode des «histoires»), mais les remarques qu'il formule sur la methodus medendi suggèrent que l'on doit aboutir à des ensembles de concepts empiriques articulés suivant une nécessité systématique, celle de l'hypothèse inductivement contrôlable. C'est ainsi que dans la médecine empiriste (de type sydenhamien) on doit progresser en direction d'une théorie physiologique qui puisse diriger la pratique («constituta alia plane corporis oeconomia» ${ }^{8}$ ). Le recours obligé aux hypothèses incite à souligner la structure spéculative de la physique chez Hobbes et Descartes. Il est clair que Leibniz a en vue une forme plus ouverte de l'hypothèse puisque les principes a priori que l'on suppose ne peuvent prétendre à une entière apodicticité. Le mode de déploiement de la théorie dans l'Hypothesis physica nova (1671) avait fourni un arrière-plan où ces considérations épistémologiques pouvaient se profiler.

Les lettres à Conring des années 1677-1678 développent avec plus de précision encore: 1) la démarcation fine par rapport à la méthodologie cartésienne des Principia; 2) la conciliation possible de la théorie définitionnelle de la science avec la doctrine «pragmatique» des hypothèses.

La physique cartésienne est une construction qui repose sur une base d'expérience insuffisante. Dans la plupart des cas, des

6. P, I, p. 174 .

7. Cf. P. I, p. 174, et surtout, p. 195 (Leibniz an Conring, 19 mars 1678).

8. P, I, p. 174 . 
observations relevant de l'expérience commune servent de seuls garants de la pertinence explicative des principes: Pris par de stériles controverses, enchaîné à son rôle de chef de secte, Descartes a dû se contenter de corrélations sommaires entre des experimenta in vulgus nota. L'analyse hypothético-déductive de type cartésien détient une puissance considérable. Certains specimens de l'oeuvre scientifique témoignent d'instances positives, par exemple les Météores et les écrits physiologiques, en particulier L'bomme et Les Passions de l'âme. Le problème du schéma cartésien est qu'à un système ingénieux d'hypothèses bâti sur des expériences aussi peu analytiques on peut constamment opposer une pluralité d'autres systèmes «adéquats». La discrimination entre les hypothèses possibles va donc dépendre d'une expérimentation qui puisse dissocier plus intimement les «compossibles» des simples possibles dans les concepts réfléchissant le divers de l'expérience. On accéderait alors à une, sorte d'apodicticité adventice qui combinerait la représentation d'un ordre causal des phénomènes et la déduction hypothétique des connexions entre phénomènes. De facto, les expériences manifestent chaque jour davantage la fragilité de la construction cartésienne en physique. Mais le projet même de cette construction semble répondre à une nécessité méthodologique certaine; l'arrangement ingénieux des éléments dans la fiction explicative peut servir de modèle à quiconque désire fonder une philosophie naturelle offrant des garanties plus complètes de pertinence objective: les instances. significatives du modèle se trouveraient dans l'explication des marées, de l'arc-en-ciel, de la génération des sels, des météores et des opérations magnétiques (phénomènes qui serviront de pierres de touche des théories jusque dans l'experimental philosophy newtotienne) ${ }^{9}$. Lorsque Leibniz caractérise les champs explorés par Descartes et les physiciens cartésiens, il présente les explications fournies comme révisables: inadéquation des lois du choc, en particulier dans le concours de deux corps durs de grandeur inégale; absence d'analyse des effets corrélatifs en chimie; utilisation insuffisante des méthodes d'expression mathématique. Prises au pied de la lettre, les hypothèses cartésiennes sont infécondes. Mais il convient en réalité de distinguer les préceptes pour l'expression mécanique et l'analyse géométri-

9. Cf. P, I, p. 186. 
que des phénomènes d'une part, les solutions hypothétiques apportées aux problèmes empiriques d'autre part.

À l'origine, il ne semble pas que Leibniz ait eu l'intention de renverser la méthodologie mécaniste à laquelle Descartes, Hobbes et Boyle se réferent. La critique s'adresse en premier lieu aux éléments de l'hypothèse physique, et Leibniz aurait défendu la formule «Omnia fieri mechanice in natura» sans réserve. Mais vers 1677-1678, l'application de la formule semble impliquer que la méthode de Leibniz se différencie de la méthode cartésienne. La notion leibnizienne d'hypothèse qui avait partie liée avec les modëles explicatifs de la physique de Descartes, tend à s'en écarter ou, pour être plus exact, à impliquer une transcription originale de la procédure hypothético-déductive. Il semble que cette refonte survient en conjonction avec l'établissement de la dynamique. De nouveaux éléments sont alors disponibles pour construire les hypothèses physiques et, tout aussi bien, pour déterminer les principes régulateurs de la procédure explicative. Dans cette perspective, une lettre postérieure à Conring sanctionne le changement de style épistémologique qui correspond à l'avènement de la dynamique.

In Physicis quoque demonstrationes aliquot habeo magni usus circa motum. Archimedes aequiponderantium scientiam constituit. Galilaeus longius progressus accelerationes sub regulam vocavit et pendulorum proprietates mirabiles detexit. Sed de vi elastica quam vocant corporum post flexionem se restituentium ac de corporum inter se concurrentium ac repercussorum legibus nunc primum certa Elementa me demonstrata habere arbitror Archimedeo plane rigore. Unde constabit non Cartesium tantum, sed et alios summos viros nondum ad intimam hujus argumenti pervenire nec proinde hactenus Scientiae mechanicae Elementa absoluta haberi ${ }^{10}$.

À la même époque, le brouillon d'une lettre destinée à Malebranche, qui ne fut jamais envoyée, témoigne d'un tournant décisif dans là critique de la physique cartésienne. Leibniz met cette fois en doute les axiomes du système cartésien: que la matière et l'étendue soient la même chose; que toute vérité dépende de l'arbitraire de la volonté divine; qu'il se conserve toujours la même quantité de mouvement dans les corps. Dans la

10. P, I, p. 202. 
même veine, il met en cause les raisons démonstratives de la loi cartésienne des sinus. Ces arguments reposaient sur l'analogie de la réfraction et des déterminations mécaniques du choc suivant le modèle cartésien. Leibniz juge l'hypothèse fallacieuse, et il étend alors sa critique à toute la physique cartésienne qu'il déclare non-explicative: "Je ne veux pas toucher à son hypothèse physique, car on ne la saurait prouver qu'en expliquant les phénomènes de la nature» ${ }^{11}$. Par exemple, les expériences de Newton font douter que Descartes ait réellement expliqué l'arcen-ciel. Et l'explication de l'aimant est déficiente pour autant qu'elle ne rend pas compte de la déclinaison. Les conceptions de Descartes sont «ingénieuses au possible, mais souvent incertaines et stériles» ${ }^{12}$. Toutefois, le rejet complet du modele cartésien est différé pour des raisons méthodologiques ou, à tout prendre, épistémologiques:

Néanmoins je conseillerais toujours à un amateur de la vérité [d'] approfondir [le système cartésien], car on y voit une adresse d'esprit admirable et sa physique tout incertaine qu'elle est, peut servir de modèle à la véritable, qui doit pour le moins être aussi claire et aussi concertée que la sienne; car un Roman peut être assez beau pour être imité par un historiographe. Pour l'abréger: Galilei excelle de réduire les mécaniques en science, des Cartes est admirable pour expliquer par de belles conjectures les raisons des effets de la nature ${ }^{13}$.

En brisant l'écorce métaphorique de ces comparaisons, ne peut-on inférer que le modèle cartésien, s'il était repensé en fonction des problèmes que soulève la compréhension empirique de la nature, représenterait une forme de démonstration apte à intégrer les données d'expérience en un système rationnel? Une autre façon d'interpréter ce passage consisterait à supposer que les explications cartésiennes sont de pures conjectures, mais qu'on peut les soumettre à une critique qui révèle les présupposés impliqués et suggère des critères plus adéquats pour la transposition analogique de données d'expérience. Pour être adéquate, cette transposition pourrait dépendre d'une analyse conceptuelle des éléments impliqués dans notre compréhension des phénomènes. Pour Leibniz, toute conjecture est susceptible d'analyse et de

11. P, I, p. 334.

12. P, I, p. 336 .

13. P, I, p. 336 . 
vérification, donc un contrôle peut en entraîner la révision. Il vaut la peine de noter qu'il souhaite la venue d'un esprit apte aux conjectures rationnelles pour hausser la médecine empiriste au rang de discipline rationnelle. Dans de semblables domaines, les hypothèses de type cartésien amorcent le développement d'arguments démonstratifs, ce qui ne peut se faire au départ que par l'anticipation hypothétique.

Il serait intéressant de reprendre, dans cette perspective, l'étude du remodelage que Leibniz envisage sur le matériau de la physique cartésienne. Le corpus disponible est considérable, en particulier si l'on veut tenir compte des rectifications qu'implique l'instauration de la dynamique. L'analyse pourrait se concentrer sur la lecture critique des Principia par Leibniz (Cf. Animadversiones in partem generalem Principiorum Cartesianorum, 1692). Ce fut une préoccupation majeure pour Leibniz, comme en témoigne l'analyse faite par Belaval d'une première version des Animadversiones remontant à $1675^{14}$. Comme les débuts de la dynamique peuvent être situés dans la première moitié de 1678, un tel ensemble de réflexions critiques se développe en conjonction avec les éléments originaux du système leibnizien de la nature et se trouve intégré à la conception des hypothèses de physique.

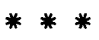

II

Or, l'amorce des doctrines épistémologiques de Leibniz ne saurait être tirée de Descartes. Certes, ces doctrines subissent une inflexion déterminante avec l'examen de la structure démonstrative et là remise en cause des modèles et présupposés cartésiens. Mais Leibniz déclare lui-même que sa divergence par rapport au programme cartésien de physique avait précédé l'étude qu'il en a faite. Ainsi s'explique-t-il à Malebranche:

Comme j'ai commencé à méditer lorsque je n'étais pas encore imbu des opinions cartésiennes, cela m'a fait entrer dans l'intérieur des choses par une autre porte et découvrir de nouveaux pays, comme les étrangers qui font le tour de France suivant la trace de 'ceux qui les ont précédés, n'apprennent presque rien

14. Yvon Belaval, 'op. 'cit., «Premières Animadversions sur les 'Principes' de Descartes», pp. 57-85. 
d'extraordinaire, à moins qu'ils soient fort exacts ou fort heureux; mais celui qui prend un chemin de traverse, même au hasard de s'égarer, pourra plus aisément rencontrer des choses inconnues aux autres voyageurs ${ }^{15}$.

Du point de vue épistémologique, ce chemin de traverse correspond sans doute à la conciliation entrevue de la théorie définitionnelle de la science et de la justification pragmatique des hypothèses. À la période cruciale où s'élabore la dynamique, un thème convergent fait son apparition alors que Leibniz critique la transposition que Foucher a opérée des arguments des sceptiques, et qu'il discute les vérités hypothétiques: «Dans le fond, toutes nos expériences ne nous assurent que de deux choses, savoir qu'il y a une liaison dans nos apparences qui nous donne le moyen de prédire avec succès des apparences futures, l'autre que cette liaison doit avoir une cause constante» ${ }^{16}$. La vérité des propositions hypothétiques est de ce fait quelque chose hors de nous, qui ne dépend pas de nous. Notre pouvoir de conceptualiser l'ordre vraisemblable de la nature phénoménale suivant les analogies de l'expérience se règle sur ce qui peut être considéré comme analytique dans le système des phénomènes, et cette considération dépend elle-même de la continuité reconstituée des raisons suffisantes particulières. Dans cette ligne, Leibniz rejette toute forme métaphysique de doute et laisse place à une critique progressive des schématismes trompeurs de l'expérience (notions confuses). D'une part, les hypothèses sont des anticipations de raisons suffisantes qui échappent à toute tentative de déploiement analytique direct. D'autre part, en établissant une explicitation analytique des hypothèses sous couvert de présupposés admissibles, comme le principe de continuité, on peut montrer qu'une hypothèse donnée a le pouvoir d'exprimer et de systématiser le divers des relations empiriques. Cette "géométrisation» de la connaissance empirique confere leur statut rationnel aux vérités hypothétiques $^{17}$.

15. Leibniz an Malebranche, 22 juin 1679, P, I, p. 332.

16. Leibniz an Foucher, P, I, p. 372.

17. Cf. P, I, p. 381: «En matière de connaissances humaines, il faut tâcher d'avancer, et quand même ce ne serait qu'en établissant beaucoup de choses sur quelque peu de suppositions, cela ne laisserait pas d'être utile, car au moins nous saurons qu'il ne nous reste qu'à prouver ce peu de suppositions pour parvenir à une pleine démonstration, et en attendant, nous aurons au moins des vérités hypothétiques, et.nous sortirons de la confusion des disputes. C'est la méthode des Géomètres...". 
Du statut des vérités hypothétiques, on peut aisément inférer et établir certaines caractéristiques des hypothèses de physique. Ainsi Leibniz concède-t-il (à Conring) que la science de la nature repose sur l'association de l'experientia et de la ratio apodictica (ou, à défaut de telles raisons, sur des raisons probables $[\delta o \xi \alpha \sigma \tau \iota \kappa \eta]$, mais sa conception de la ratio apodictica va lui permettre de fixer le modèle épistémologique pour une théorie des hypothèses bien formées:

Ego semper putavi, Demonstrationem nihil aliud esse quam catenam definitionum vel pro definitionibus, propositionum jam ante ex definitionibus demonstratarum aut certe assumtarum. Analysis autem nihil aliud est quam resolutio definiti in definitionem, aut propositionis in suam demonstrationem, aut problematis in suam effectionem. Sed quando plures ejusdem rei effectiones fingi possunt, tunc nova quaerenda sunt data sive experimenta, quibus excludantur eae effectiones sive causae quae non sunt hujus loci. Si vero ejusmodi data nova (qualia Verulamius instantias crucis vocat) non sint in promptu, tunc non possumus praecise designare causam effectus veram, sed contenti esse cogimur hypothesi sive causa possibili, quae quo simplicior et concinnior, hoc probabilior, ut in Astronomicis Copernicana, in quibusdam Physicis Cartesiana ${ }^{18}$.

Il est caractéristique que le modèle de la "chaîne de définitions» se prête à des schèmes de suppléance en faveur de chaînes de propositions où interviennent des sortes d'éléments premiers présumés avec certitude. La garantie de la démonstration conçue suivant ce modèle réside dans la possibilité d'effectuer l'analyse des chaînons dérivés. Cela s'étend à la résolution des problèmes par une analyse qui remonte à des conditions suffisantes. S'il y a une alternative de solutions possibles, la difficulté peut être surmontée en étendant l'analyse de façon à y inclure des instances cruciales susceptibles de diriger le développement analytique vers l'une des branches. Ces instances spécifieraient plus complètement les conditions empiriques du problème: aux termes initiaux du problème s'ajouteraient les termes complémentaires déterminant l'expérience cruciale. Leibniz, semble-t-il, distingue deux types de résolutions de problèmes complexes: 1) les solutions que l'on peut compléter analytiquement par des expériences discriminant entre les raisons suffisantes de façon de plus en plus adéquate: on se rapproche alors des chaînes démonstratives par

18. Leibniz an Conring, 3 janv. 1678, P, I, p. 186. 
substitution d'équivalents; 2) les solutions qui se limitent à conjecturer les conditions déterminantes des faits disponibles (cas de l'hypothèse copernicienne ou des hypothèses physiques de Descartes): les éléments du problème sont alors retranscrits suivant un ordre présumé. Cette deuxième forme de résolution ne peut certes prétendre à la vérité apodictique, mais on peut lui accorder un degré de probabilité correspondant à sa simplicité ou à son élégance formelle (cohérence de l'ordre figuré par l'explicatum). A l'occasion, Leibniz suggère que ces hypothèses du deuxième ordre pouvant donner lieu à des alternatives dans l'explication, une détermination de probabilités par le calcul pourrait rattacher l'investigation au modèle définitionnel de la démonstration $^{19}$. Mais il reconnaît que cette pratique n'en est qu'à ses débuts $^{20}$.

Le principal problème relatif à la résolution analytique par voie d'hypothèse consiste dans le fait qu'un explicans satisfaisant aux conditions de l'explicandum peut être inféré de principes faux. Conring souligne l'obstacle avec force: "Nova data quin possint effingi atque ex iis utut faisis aliquid demonstrari, nemo negaverit. Sed ejusmodi demonstrationes non tamen sunt $\epsilon \pi \iota \sigma \tau \eta \mu o \nu \iota$ $\kappa \alpha \iota$, nec per analysin colligere licet datorum veritatem ex veritate consequentium» 21 . Conring englobe dans sa critique tant les hypothèses cartésiennes que les procédures inférentielles que l'on peut rattacher à l'experimental philosophy. Toutefois, Leibniz avait spécifié les conditions formelles de validité pour la procédure analytique: la chaîne des propositions doit impliquer des arguments réciproques, c'est-à-dire des propositions dont la converse soit également vraie, à l'instar des définitions - sans nul doute

19. Cf. Nouveaux èssais sur l'entendement bumain, 4. 17.3: «[Les] liaisons [des vérités] sont même nécessaires quand elles ne produisent qu'une opinion, lorsque après une exacte recherche la prévalence de la probabilité, autant qu'on en peut juger, peut être démontrée, de sorte qu'il y a demonstration alors, non pas de la vérité de la chose, mais du parti que la prudence veut qu'on prenne». (P, V, p. 457).

20. Cf. N.E. , 4.16.9: «J'ai dit plus d'une fois qu'il faudrait une nouvelle espèce de logique, qui traiterait des degrés de probabilité, puisqu'Aristote dans ses Topiques n'a rien moins fait que cela, et s'est contenté de mettre en quelque ordre certaines règles populaires, distribuées selon les lieux communs, qui peuvent servir dans quelque occasion, où il s'agit d'amplifier le discours et de lui donner apparence, sans se mettre en peine de nous donner une balance nécessaire pour peser les apparences et pour former là-dessus un jugement solide. Il serait bon que celui qui voudrait traiter cette matière, poursuivît l'examen des jẹu de hasard..." (P, V, p. 448).

21. Conring an Leibniz, 26 fév. 1678, P. I, pp. 190-191. 
s'agit-il des définitions réelles, qui établissent la possibilité de leur objet — et à l'instar des équations. La vérité de ce qui est démontrable tient non à des fondements d'induction, mais à la substitution d'équivalents conceptuels dans la corrélation des termes. Cela signifie l'usage des notions dont l'adéquation paraisse de façon distincte. Les propositions indémontrables au terme de la réduction analytique seront soit des propositions identiques, soit des propositions empiriques. Dans les deux cas, on a affaire à des connexions de notions (identité ou corrélation conforme au constat empirique) dont la possibilité s'établit d'emblée.

Lorsqu'en réplique à Conring Leibniz défend sa conception de l'analyse et de la démonstration, il affirme qu'elle traduit de façon congruente la pratique des savants et qu'elle indique la méthode «ad inveniendum atque judicandum». ${ }^{22}$. Le principe de validité de toute la procédure est ainsi formulé.

Patet... omnes veritates resolvi in definitiones, propositiones identicas et experimenta (quanquam veritates pure intelligibiles experimentis non indigeant) et perfecta resolutione facta apparere, quod catena demonstrandi ab identicis propositionibus vel experimentis incipiat, in conclusionem desinat, definitionum autem interventu principia conclusioni connectantur, atque hoc sensu dixeram Demonstrationem esse catenam definitionum ${ }^{23}$.

Leibniz conçoit donc un mode de démonstration hypothétique où les éléments de résolution de l'explicandum physique sont fournis par des propositions empiriques. Même si les termes de ces propositions' restent irréductiblement problématiques, cela ne saurait engendrer d'aporie en ce qui concerne la procédure démonstrative elle-même. Celle-ci ne consiste-t-elle pas dans la complète analyticité des connexions propositionnelles, qui à son tour devrait se réduire à la complète analyticité des connexions conceptuelles? Il est significatif que Leibniz établisse un double parallélisme entre résolution des idées, démonstration, c'està-dire réduction analytique des propositions à des vérités déjà connues, et résolution des problèmes en problèmes plus faciles tels qu'il soit en notre pouvoir de les résoudre. Avec la typologie des idées que l'on trouve par exemple dans les Meditationes et dans le Discours de métaphysique, on peut se représenter la gradation dans les formes de l'idée distincte comme une série de stades dans la

22. Leibniz an Conring, 19 mars 1678, P, I, p. 194.

23. Ibid., p. 194. 
progression analytique. Or, une idée claire distincte inadéquate peut donner lieu à des opérations combinatoires (et donc à des procédures démonstratives) pourvu que les notions y figurant soient soumises à une expression symbolique qui permette des substitutions d'équivalents. Si l'analyse est provisionnelle, une telle idée et ses équivalents pourraient être tenus pour adéquats pour fin de démonstration. Il est alors convenu qu'on se réserve de contrôler les éléments conceptuels impliqués sur les données d'expérience. La symbolisation et les opérations que permet le recours aux symboles instituent les conditions d'une adéquation provisionnelle et rendent possibles certaines résolutions problématiques. La typologie leibnizienne des idées vise à révéler les apories de l'intuition portant sur les notions complexes. En fait, Leibniz met en cause l'évidence cartésienne pour le motif qu'elle entraîne l'illusion psychologique d'une fausse clarté dans les connexions conceptuelles. De même, l'analyse épistémologique des hypothèses révèle qu'une hypothèse sans démonstration n'a pas de valeur bien qu'on puisse en dériver des phénomènes vérifiables (contrôle par l'observation). Entre l'explicandum et l'explicatum, la connexion doit être opérée par propositions convertibles ou par le développement d'équations car le principe logique gouvernant la démonstration stipule que des équivalents conceptuels soient substitués les uns aux autres. Certes, il reste le cas d'un principe ou d'une hypothèse (de type cartésien) qui ne peut satisfaire adéquatement à la condition d'analyticité. La simple dérivation à partir des phénomènes observés ne saurait suffire à en garantir la vérité. Aussi Leibniz renforce-t-il les critères cartésiens qui sous-tendent la transformation de la certitude morale des hypothèses en certitude physique, et il modifie la signification et la portée de l'opération.

Illud... fatendum est, hypothesin tanto fieri probabiliorem quanto intellectu simplicior, virtute autem ac potestate amplior est, id est quo plura phaenomena et quo paucioribus assumtis solvi possunt. Et contingere potest ut hypothesis aliqua haberi possit pro physice certa, quando scilicet omnibus omnino phaenomenis occurrentibus satisfacit, quemadmodum clavis in cryptographicis. Maxima autem (post veritatem) laus est hypotheseos, si ejus ope institui possint praedictiones, etiam de phaenomenis seu experimentis nondum testatis, tunc enim in praxi hypothesis ejusmodi pro veritate adhiberi potest ${ }^{24}$.

24. Ibid., pp. 194-195. 
Ce texte fait évidemment référence à Principia , IV, $\$ \S 203-206$, où Descartes justifie son système d'hypothèses en montrant qu'il se conforme aux premières notions intelligibles représentant la nature corporelle pour une connaissance a priori. La fiction de parties matérielles imperceptibles auxquelles on attribue grandeurs, figures et mouvements appropriés, suffirait à fournir une explication des phénomènes perceptibles. D'autres modes de production des phénomènes sont sans doute possibles. Cette hypothèse n'a donc qu'une valeur pratique (sufficit ad usum vitae); et cela suffit, pourvu qu'elle corresponde exactement à tous les phénomènes pertinents. La certitude morale qui s'y rattache, est du même ordre que celle qui découle d'avoir inventé une grille pour traduire le sens supposé d'un cryptogramme. La vérité de l'interprétation est proportionnelle à la persuasion fondée qui découle du fait que les phénomènes ont été réunis en une représentation cohérente. Cette certitude morale des hypothèses corroborées pourrait être en outre garantie par référence à la véracité divine, puisque ce principe métaphysique garantit l'application du critère de l'évidence dans les démonstrations, y compris dans les inférences hypothético-déductives. La connaissance de l'existence des réalités matérielles, comme d'ailleurs l'ensemble des arguments qui établissent le système de la nature, relève de l'évidence par le lien de la cohérence déductive et/ou par l'instruction provenant de la «lumière naturelle» (suivant les dispositifs inscrits dans notre nature $)^{25}$.

Or, Leibniz se dissocie en fait de certains aspects de la doctrine cartésienne, en donnant, semble-t-il, une autre orientation à l'analyse. En premier lieu, il n'y a pas de raison de devoir recourir à la métaphysique pour établir l'objectivité des hypothèses particulières. Les preuves interviennent de l'intérieur même de l'argumentation hypothétique s'il est fait adéquatement usage des hypothèses: la probabilité de l'hypothèse (suivant une gradation continue dans l'approximation de la vérité, ce qui n'aurait aucun sens pour Descartes) repose sur la simplicité de l'explicans jointe à l'ampleur de sa conformité aux faits. Le déploiement de rationalité dans l'explicans objective un ordre virtuel des données; si l'analyse devenait complète et adéquate, elle nous rapprocherait asymptotiquement de l'ordre réel. La certitude morale débouche

25. Cf. Descartes, Principia philosophiae, IV, \$ 206, A.T., VIII-I, pp. 328-329. 
de plein droit sur la certitude physique et exprime donc les «raisons» de la nature pourvu que l'hypothèse satisfasse à toutes les déterminations d'expérience. Mais le trait le plus significatif tient au rôle heuristique dévolu à l'hypothèse. Cette fonction, lorsqu'elle est adéquatement remplie, transforme la certitude physique en une sorte de vérité. Il en est ainsi parce que le déploiement analytique des termes constituant l'hypothèse, donne forme au divers des phénomènes suivant la nécessité présumée de principes architectoniques, tel le principe de continuité. Au sens strict, la véracité divine qui sert de caution de vérité dans le système de Descartes, est un deux ex machina stérile en comparaison du pouvoir d'analyse que recèle une hypothèse leibnizienne et qu'elle peut déployer en inventant des explicata bien structurés. Et l'on doit ajouter que le critère d'adéquation pour les notions complexes ne consiste plus dans la clarté trompeuse d'une représentation nécessairement partielle, limitée, tronquée, mais il consiste désormais dans le pouvoir d'articuler les implications logiques de concepts que l'on a forgés pour rendre compte de connexions non encore explorées dans le monde des phénomènes; il consiste, corrélativement, dans la capacité de lier les éléments de la représentation en un système de la nature progressivement révisable.

Par sa conception de l'hypothèse de physique, Leibniz entrevoit donc une stratégie d'analyse qui porterait sur des «construits» représentant l'analogie de plus en plus exacte des phénomènes significatifs. Cette notion d'hypothèse, notion d'une variante complexe d'argumentation "géométrique», est, comme vous l'aurez constaté, un concept épistémologique, et peut-être même une simple représentation épistémologique. Dans son abstraction, elle ne saurait sans doute déterminer aucun travail effectif d'explication des phénomènes.

Et pourtant, j'aimerais suggérer qu'elle éclaire certaines instances paradigmatiques de la pratique leibnizienne en science: par exemple, la nécessité, en dynamique, de retranscrire la démonstration a posteriori des théorèmes de conservation en démonstration a priori. Pour Guéroult, il s'agit d'un piège cartésien où Leibniz 
s'enferre inutilement, car la dynamique devient incohérente lorsqu'elle est coupée de ses présupposés monadologiques ${ }^{26}$. A mon avis, ce serait une démarche obligée pour que la procédure hypothétique se justifie comme analyse et acquière le maximum d'autonomie par rapport aux principes métaphysiques qui sont d'un autre ordre. Guéroult y voit la mise en échec du système. Mais les démonistrations mathématiques sont-elles assignables à l'intérieur du système? Pourquoi les hypothèses de physique devraient-elles être circonscrites avec rigueur par les concepts d'un système métaphysique, puisque l'impératif véritable est de tendre à l'expression la plus analytique tout en rendant compte des séquences de phénomènes? L'alternative des deux voies de démonstration en mécanique illustre la relative équivocité du statut d'hypothèse dans la science leibnizienne. Mais il en serait de même en dioptrique, alors que Leibniz combine ingénieusement - mais l'artifice efface-t-il toute ambiguïté? - telle conjecture, relative aux effets mécaniques des milieux sur le rayon réfracté (vitesse proportionnelle et diffusion inversement proportionnelle à la densité du milieu) et telle expression géométrique du phénomène permettant d'assigner par analyse (calcul de maximis et minimis) les coordonnées d'un déplacement angulaire optimal (dans les limites de la conjecture) ${ }^{27}$.

L'hypothèse fournit des succédanés d'argumentation apodictique dont la qualité immédiate est d'ordonner les phénomènes en séquences intelligibles: ce qui facilite l'invention rationnelle (validité heuristique). Mais l'hypothèse doit tendre à la justification analytique: ce qui la subordonne à la possibilité d'une transcription géométrique, si l'on veut comprendre sous ces termes toute technique apte à fixer l'explicans en une expression suffisamment analytique. Une hypothèse bien formée est alors invention d'analyticité par substitution d'équivalents (validité démonstrative).

Département de philosophie Université de Montréal

26. Cf: Martial Guéroult, Leibniz. Dynamique et Métaphysique. 'Paris: Aubier-Montaigne, 1967, chap. V, pp. 110-154.

27. Cf. F. Duchesneau, "Hypothèses et finalité dans la science leibnizienne", Studia leibnitiana, XII (1980), pp. 161-178. 